Meta

Journal des tradlucteurs

Translators' Journal

\title{
Le tableau de bord de l'automobile
}

\section{Arthur Bédard et Marcel Marquis}

Volume 15, numéro 4, décembre 1970

URI : https://id.erudit.org/iderudit/003582ar

DOI : https://doi.org/10.7202/003582ar

Aller au sommaire du numéro

Éditeur(s)

Les Presses de l'Université de Montréal

ISSN

0026-0452 (imprimé)

1492-1421 (numérique)

Découvrir la revue

Citer cet article

Bédard, A. \& Marquis, M. (1970). Le tableau de bord de l'automobile. Meta, 15(4), 231-235. https://doi.org/10.7202/003582ar d'utilisation que vous pouvez consulter en ligne.

https://apropos.erudit.org/fr/usagers/politique-dutilisation/ 


\section{LE TABLEAU DE BORD DE LAUTOMOBILE}

Les nombreux dispositifs et appareils installés dans les voitures du dernier cri causent souvent au traducteur et au rédacteur des problèmes linguistiques sans nombre. Conduire une automobile est chose facile, mais il n'en va pas de même

34. Commonwealth Universities Yearbook, 1967, p. 179.

35. Calendar of the Teachers' College $1967 / 1968$, Toronto, Ontario Dept. of Education, 1968 , p. 23.

36. Voir : États-Unis.

37. Commonwealth Universities Yearbook, 1967, p. 520.

38. Ibid.

39. Horizons, Toronto, Ontario Dept. of University Affairs, 1967, p. 21. 
lorsqu'il s'agit de décrire les dispositifs et appareils de la voiture. Commençons donc notre petit inventaire terminologique par le début, c'est-à-dire le démarrage.

Le commutateur d'allumage (ignition switch) comprend de nos jours l'antivol (anti-theft switch), le contact (switch on) et la commande du démarreur (starter). Donner un nom à cet ensemble n'est pas chose facile ; en faisant abstraction d'un élément - le démarreur — il est possible de l'appeler un antivol contact, comme le font les fiches descriptives Esso, un peu sur le modèle de contact démarreur. Toutefois, on ne peut guère l'appeler un antivol contact démarreur, si ce n'est que de façon très descriptive dans un guide de l'automobiliste, par exemple.

Par temps froid, une automobile se refuse souvent à démarrer. Un dispositif, soit automatique, soit manuel (tirette - pull knob), est donc prévu pour enrichir le mélange d'essence et d'air et faciliter l'allumage. Les Français ont emprunté à tort le terme anglais starter pour décrire ce que les Anglais, eux, appellent choke. $\mathrm{Au}$ Canada français, le terme étrangleur, traduction littérale, retint tout d'abord l'attention. Il n'eut cependant pas l'heur de plaire à certains puisque le Comité d'étude des termes techniques français proposa enrichisseur, un néologisme certes de bon aloi et fort exact du point de vue descriptif. Une nouvelle expression, assez jolie d'ailleurs, est récemment venue s'ajouter aux précédentes : on parle maintenant d'un volet de départ.

L'avertisseur sonore (horn), ou klaxon (à noter qu'il s'agit là d'une marque déposée), étant souvent interdit dans les villes, les fabricants ont maintenant installé sur certaines voitures européennes un avertisseur optique (headlamp flasher). Une commande, généralement placée sur la colonne de direction, permet, par une simple pression du doigt, de donner un appel de phares avertisseur.

Les phares (headlights) ont droit à une double commande. Le commutateur d'éclairage (headlights switch) sert, comme son nom l'indique, à allumer les phares. Le commutateur de code (dimmer switch) permet de choisir soit le faisceau route (high beams), soit le faisceau code (low beams). Ajoutons que les expressions suivantes sont aussi en usage : feux de route, phares-route, faisceau de croisement, feux de croisement, phares-code. Il faut toutefois signaler que le terme "feux » est normalement utilisé pour parler des lampes de position et non de l'éclairage d'un véhicule.

Les clignotants (turn signals) sont commandés par un inverseur de courant (reversing device) installé sur la colonne de direction et servent à indiquer l'intention de faire un virage. Il ne faut pas les confondre avec le clignoteur, petit appareil qui fait fonctionner les clignotants. Un autre inverseur commande le système multiclignotant (four-way hazard warning flasher), dispositif qui fait fonctionner les quatre clignotants à la fois. On l'utilise pour indiquer le danger, par exemple en cas de panne.

De nouvelles inventions viennent s'ajouter sans cesse aux déjà très nombreux accessoires offerts « en supplément ». La minuterie d'extinction des phares (light watch system) est un dispositif qui, une fois déclenché, garde les phares allumés pendant une trentaine de secondes. Il peut se révéler des plus utiles pour se rendre, par exemple, de l'automobile à la maison par une nuit d'encre. Le lave-phares 
(headlamp washer) nettoie les phares grâce à un jet d'eau sous pression. L'essuiephares (headlamp wiper) vient d'être mis sur le marché. Il s'agit d'une brosse en forme d'hélice montée sur pivot; fixée sur la surface du phare, elle est actionnée par le vent lorsque la voiture se met en route.

Les grosses voitures peuvent aussi être équipées d'un régulateur de vitesse (automatic speed control) et d'un volant inclinable (tilt steering wheel). Le premier appareil maintient la voiture à la vitesse choisie par le conducteur sans que celuici ait à garder le pied sur l'accélérateur. Il se désengage lorsque le conducteur touche les freins. Le volant inclinable, commandé par un levier de dégagement (unlocking lever), est monté sur pivots et peut être déplacé verticalement — parfois horizontalement - afin de faciliter l'entrée et la sortie du conducteur et lui permettre d'adopter une position au volant aussi confortable que possible.

Le changement de vitesse peut se faire manuellement ou automatiquement. Dans le cas de la boîte manuelle, on parle d'un levier de changement de vitesse ou d'un levier de vitesse (gear change lever) et dans le cas d'une transmission automatique, d'un levier sélecteur de vitesse (selector lever), ou sélecteur. Dans le deuxième cas, on trouve sur le tableau de bord ou sur la colonne de direction le secteur (quadrant) où sont imprimées les abréviations des différentes vitesses. L'index du sélecteur (selector index), sorte de stylet, indique le rapport choisi par le conducteur. Les équivalents français des abréviations anglaises $P R N D L$ sont, par ordre : $\mathbf{P}$, parking, $\mathbf{A R}$, marche arrière, $\mathbf{N}$, neutre, $\mathbf{V R}$, ville-route et $\mathbf{E}$, exceptionnelle.

Le levier de changement de vitesse, ou le sélecteur, est installé soit sur la colonne de direction (column), soit sur le bloc de commande (console). Certains modèles, toutefois, sont équipés d'un levier au plancher (stick shift), le bloc de commande étant situé plus vers l'avant.

Le terme «compteur » s'emploie de multiples façons : compteur kilométrique (kilometric recorder), compteur totalisateur (total mileage indicator), compteur journalier (trip recorder, daily mileage indicator) et même "compteur de vitesse " (speedometer). Cette dernière expression est toutefois impropre. En effet, l'appareil en question ne compte pas la vitesse du véhicule, il l'indique; il faut donc parler d'un indicateur de vitesse. Le terme correspondant à compteur kilométrique ne semble pas en usage à l'heure actuelle au Canada. Bien qu'un constructeur nous propose compteur de milles, nous préférerions compteur milliaire (mileage indicator). L'adjectif « milliaire » existe déjà en français et se rapporte au mille romain. Il ne s'agirait donc que d'une légère extension de sens.

Le même constructeur nous propose aussi un terme générique pour tous les appareils de calcul des distances : odomètre. À l'origine, ce terme était utilisé comme synonyme de «podomètre » et de « compte-pas », appareils qui servaient à calculer une distance parcourue à pied. Toutefois, ce terme est une composition savante à partir des mots grecs hodos (route) et metron (mesure). L'extension de sens semble donc justifiable.

Dans les régions où le climat est tempéré, les automobiles sont équipées d'appareils de chauffage, de dégivrage et de désembuage. En France, la question 
en est restée là du point de vue linguistique ; on parle de la commande du chauffage, du dégivrage, du désembuage. Au Canada, cependant, les termes chaufferette (heater), dégivreur (defroster) et désembueur (demist) sont entrés dans la langue. Les deux usages sont linguistiquement exacts. Seul le temps saura décider de la norme à suivre.

MINI-GLOSSAIRE

DU TABLEAU DE BORD DE LAUTOMOBILE

air intake shut control

ammeter

anti-theft switch

ashtray

automatic speed control

blower

charge indicator

choke

cigarette-lighter

column

console

courtesy lights

dashboard

defogger (demist)

defogger (rear-window...)

defroster

dimmer switch

electric clock

four-way hazard warning flasher

fuel gauge

fuses

gas pedal

glove compartment (...box)

handbrake

headlamp flasher

headlamp switch, headlight switch

headlamp washer

headlamp wiper

heater

high beams

horn

horn button

horn circle (...ring)

horn (town and country...)

ignition switch

light watch system

low beams

mileage indicator

mileage indicator (daily...)

mileage indicator (total...)

oil pressure gauge

overdrive

power brake

power steering

pull knob

quadrant

radio set fermeture d'admission d'air

ampèremètre

(dispositif) antivol

(générique) antivol contact démarreur

cendrier

régulateur de vitesse

soufflerie

indicateur de charge

starter (fr.), étrangleur (can.),

enrichisseur, volet de départ

allume-cigarette, allume-cigare

colonne de direction

bloc de commande

lampes d'accueil

tableau de bord, planche de bord

désembuage (fr.), désembueur (can.)

désembueur de lunette

dégivrage (fr.), dégivreur (can.)

commutateur de code

montre électrique

système multiclignotant

indicateur d'essence, jauge d'essence

fusibles

accélérateur

boîte à gants

frein à main

avertisseur optique

commutateur d'éclairage

lave-phares

essuie-phares

chaulfage (fr.), chaufferette (can.)

faisceau route, phares-route,

" feux de route?

avertisseur sonore, klaxon

bouton d'avertisseur

cerclo-avertisseur

avertisseur ville et route

commutateur d'allumage, contact d'allumage

minuterie d'extinction des phares

faisceau code, phares-code,

faisceau de croisement,

" feux de croisement "

odomètre

compteur journalier

compteur totalisateur

indicateur de pression d'huile,

manomètre

surmultiplication

freins assistés, servo-freins

direction assistée, servo-direction

tirette (du volet de départ)

secteur

(poste) radio 


\author{
rearview mirror \\ selector lever \\ shift lever \\ speedometer \\ stick shift \\ sun-visor \\ tachometer \\ tilt steering wheel \\ turn signals \\ turn signals lever \\ unlocking lever \\ ventilation hole \\ voltmeter \\ warning light \\ windscreen washer \\ windscreen wiper
}

rétroviseur

levier sélecteur de vitesse, sélecteur

levier de changement de vitesse,

levier de vitesse

indicateur de vitesse

levier de vitesse au plancher

pare-soleil

tachymètre (compte-tours)

volant inclinable

clignotants

inverseur des clignotants

levier de dégagement

buse d'aération, bouche d'air, diffuseur

voltmètre

témoin de..., voyant

lave-glace, lave-vitre

essuie-glace, essuie-vitre.

ARThur Bédard et Marcel Marquis 